\title{
ABC-Based Optimization of Cluster Head Selection in Wireless Sensor Networks
}

\author{
Sulaiman M. Karim ${ }^{1}$, Celal Ozturk ${ }^{2}$ and Musaria K. Mahmood ${ }^{3}$ \\ ${ }^{1}$ Department of Computer Engineering, Karabuk University, Karabuk, Turkey \\ ${ }^{2}$ Department of Computer Engineering, Erciyes University, Kayseri, Turkey \\ ${ }^{3}$ Department of Electrical and Electronics Engineering, Istanbul Gelisim University, Istanbul, Turkey \\ suleymankerim@ogrenci.karabuk.edu.tr,mkmahmood@gelisim.edu.tr
}

Abstract: There are several advantages of using Wireless Sensor Networks (WSNs) compared to wired sensor networks, such as lower cost, easier deployment, and better scalability. WSNs are employed in many fields such as medicine, industry, and economics, among others. As the use of WSNs grows in a number of applications, further work is being done on research and development of new methods to enhance their performance. Selecting the proper routing protocol is a significant consideration and an important task for all WSN applications. Many pieces of research focused on the routing problem which can contribute to the improvement of WSN lifetime and problem-solving techniques. This paper, however, discusses the various existing optimization techniques, including numerous modifications to the LEACH protocol. It then conducts a series of simulations to notice the difference between utilizing LEACH alone and $\mathrm{LEACH}$ with the $\mathrm{ABC}$ optimization algorithm. The purpose of these experiments is to prove the improvement made by the $\mathrm{ABC}$ on the residual energy which is reflected positively in the number of received packets and minimize the dead nodes. This will demonstrate how to optimize WSN system towards energy conservation. LEACH with ABC revealed much better results than LEACH on its own. This indicates one possible optimization technique that limits dead nodes and increases overall residual energy usage and the network lifetime. Many scenarios are simulated to validate the improvement of the WSN performances.

Keywords: WSN; LEACH; ABC; optimization

\section{Introduction}

Wireless Sensor Network (WSN) consists of a group of sensors (called nodes), deployed over a specific area for data collection and monitoring of physical phenomena. Collected data are transferred by wireless communication and classified in a central node called the Base Station (BS). WSNs are used for physical condition evaluation such as pollution, temperature, noise, flooding, and wind speed. WSN may be a group of hundreds or thousands of nodes connected in certain topologies depending on the type of measured physical phenomena and supervised area [1]. WSNs were originally developed for military applications such as detecting enemy forces and monitoring demilitarized zones as strategic war tools [2]. Industrial applications of WSN are unlimited since each day a new application appears in the market, especially with the era of the Internet of Things [3-4]. WSNs are used in medical applications as a subsystem for enhancing disease detection as well as monitoring the health status of patients inside and outside hospitals [5]. WSNs are also present in many agriculture and environment applications such as measuring water level, soil moisture in the field, flooding, and weather prediction [6]. WSN communicates over a wireless channel with the capability of performing a simple processing operations on the collected data and transmitting them from node-to-node to the BS. Due to their low-price and low-complexity, sensors are characterized by their short transmission range, poor processing competence, low data rates, low on-chip memory, and limited available supply. Since WSNs are self-contained systems, energy management is essential for devices. The energy stored in batteries inside sensors is consumed by the information transmission from node to node. Wireless transmission requires a certain level of energy to perform data circulation correctly depending on the distance between nodes and the amount of data exchanged. Routing data across WSN is

Received: April $5^{\text {th }}, 2020$. Accepted: May $26^{\text {th }}, 2021$

DOI: $10.15676 /$ ijeei.2021.13.2.3 
then the principal source of energy consumption. Routing is one of the critical tasks in WSNs, and because of their fundamental characteristics, they are challenging to work with in any application[7]. Based on network architecture, routing protocols in the WSNs can be classified into two classes: flat routing and clustering routing. Due to various advantages, clustering routing has become an attractive class of routing strategy in WSNs [8]. Clustering is one of the techniques used to efficiently manage node energy. In this structure, a single-layer network is used to decrease power consumption by holding the sensor efficiently to engage it in a two-hop connection within groups. Cluster routing mechanisms are more accurate and preferable for WSN with continuous data flow [9]. The Low-Energy Adaptive Clustering Hierarchy (LEACH) protocol is a TDMA-based protocol integrated with clustering and a simple routing protocol in WSNs. LEACH is a protocol that combines the notion of energy efficiency cluster-based routing together with data aggregation to achieve scalable routing and fair media access with improved performance in system lifetime [10]. Artificial Bee Colony (ABC) is one of the most promising optimization techniques in this field. It is founded on the biological process natural conduct of real honey bees in food foraging. Moreover, $\mathrm{ABC}$ algorithm has a lot of advantages such as simplicity, flexibility and robustness [11]. The aim of this research is to conduct a comparative study on the performances of LEACH and $\mathrm{ABC}$ protocols focusing on the problem of optimal selection of cluster head $(\mathrm{CH})$ in WSNs.

The article is organized as follows: the state of art is reviewed in Section 2; the theoritical information about $\mathrm{LEACH}$ and $\mathrm{ABC}$ are presented in Section 3; To illustrate the improved performance of the application of $\mathrm{ABC}$ for $\mathrm{CH}$ selection, the simulation of two case studies is given in Section 4; Section 5 concludes the article.

\section{Literature Survey}

The present research has three components: the WSN routing protocols, LEACH protocols, and the application of $\mathrm{ABC}$ to optimally resolve the problem of $\mathrm{CH}$ selection. A limited survey is conducted according to the three overlapping components taking into account the importance and the recentness of published research. A dynamic selection of $\mathrm{CH}$ is presented in [12], where cluster nodes select the next CHs depending on that residual energy of all cluster nodes. An energy-efficient protocol for heterogeneous WSN based on clustering technique is proposed by [13]. To increase the WSN lifetime, the protocol uses channel state as a function for $\mathrm{CHs}$ selection. Simulation proved the effectiveness of the proposed algorithm in stability compared to a classical protocol such as LEACH, and SEP. The relationship between sensor-node energy and the method of choosing the best $\mathrm{CH}$ candidate through the suggestion of a novel protocol has been investigated in [14]. The proposed method creates a dynamic cluster employing secure sensor protocols for information via negotiation where its drawbacks are controlled. Many routing algorithms, and data transmission protocols have been proposed for WSNs where energy sensibility is the major design issue. Many kinds of clustering protocols have been designed to balance and optimize the sensor node lifetime. LEACH protocol based on clustering protocol with randomized rotation of $\mathrm{CHs}$ has been proposed in [15]. It uses localized coordination and is able to construct scalable, robust, and dynamic networks. A novel protocol for data routing in WSN named the Energy Efficient LEACH (EE-LEACH) has been introduced in [16]. The proposed protocol offers an energy-efficient routing strategy for WSN based on efficient data gathering and optimum clustering. The importance of WSNs energy management is remarked in [17], where a novel protocol is proposed based on LEACH. A simulation of various parameters by MATLAB has been done to validate the good performances of the new protocol. Threshold equations are used for the energy evaluation of all sensors. A cluster-based scheme is proposed as a solution for the energy extension. An Extended High Energy First (EHEF) clustering protocol is proposed in [18] which is able to perform multi-hop transmissions. It is a Multi-Input Multi-Output (MIMO) scheme extending by $75 \%$ the stand-alive nodes in the network compared to the normal LEACH. A general survey of LEACH variants-based routing protocols is presented in [19] and the improvement of each research is outlined. 
The ABC algorithm has been first proposed by Karaboga at Erciyes University (Turkey) in 2005 , which could simulate the behavior of honey bees in real life and is used as an optimization technique [20]. The colony of artificial bees is modeled by three groups of bees: employed bees, onlookers, and scouts where for every food source, the model considers only one employed bee. $\mathrm{ABC}$ is a novel, robust optimization technique that can be used to find the optimal solution for a variety of engineering problems. The use of $\mathrm{ABC}$ has been first explored in a significant review to solve the clustering analysis problem in [21]. The CHs election based on the Particle Swarm Optimization technique has been studied in [22]. The proposed method is showing improved performance compared with the LEACH method. A novel clustering routing protocol based on $\mathrm{ABC}$ to find the best $\mathrm{CH}$ candidate is proposed in [23]. This algorithm is an election process of the optimal $\mathrm{CH}$ and assistant $\mathrm{CH}$ by introducing assistant $\mathrm{CH}$ in the cluster and $\mathrm{ABC}$. $\mathrm{A}$ clustering algorithm for an efficient cluster mechanism with improved $\mathrm{CH}$ selection based on a multi-objective $\mathrm{ABC}$ function is presented in [24]. An evaluation and comparison of the proposed algorithm against the existing well-known Swarm-intelligent-based techniques proves its superiority compared to others in terms of packet delivery ratio, average energy consumed, average throughput, and network life. A trust-based $\mathrm{CH}$ election protocol for multi-hop WSNs using $\mathrm{ABC}$ Algorithm is suggested in [25]. CHs usually consume their energy soon, so a mechanism for dynamic change of $\mathrm{CHs}$ is proven to be a positive step toward uniform energy utilization. An important survey about the new aspect of the analysis of routing in WSN that had never been considered in the previously available literature is presented in [26]. This work can be a guideline for future researchers to understand where we are today in the WSN technology, routing with optimized energy consumption, and the factors that have a direct impact on the energy in WSN.

\section{Theoretical Backgrounds}

\section{A. LEACH Protocol}

LEACH stands from Low-Energy Adaptive Clustering Hierarchy and is first introduced by W. Heinzelman et al. in 2000 [27]. LEACH is a cluster-based routing algorithm with the capability of auto adaptation and auto organization. The major goal of LEACH is to improve WSN lifetime. CHs are chosen in an ordered way after separating WSN into many clusters. This process presents the central concept of the LEACH protocol. Given that the consumed energy for $\mathrm{CH}$ is greater than that for non- $\mathrm{CH}$ nodes, different nodes are selected as $\mathrm{CH}$ successively balancing the consumption of energy within all nodes [15]. Data are collected from cluster nodes by the $\mathrm{CH}$ and then passed to BS in one or more hops. $\mathrm{LEACH}$ is an iterative algorithm where each round is composed of two steps: setup and steady states as shown in Fig. 1 [8]. The algorithm starts by examining the power of the sensor node, as the node is considered dead if its energy falls below a predetermined threshold. If the number of live nodes reaches a minimum (according to the application), then WSN will be out of service.

- Step stage: -

A distributed algorithm without the requirement of possible communication with the BS is used for cluster formation. This algorithm selects $\mathrm{CH}$ and distributes its location to all other nodes. At each round, nodes use a random probabilistic algorithm to determine whether they will become a $\mathrm{CH}$. Three sub-stages constitute the setup stage. They are announcement, cluster formation, and transmission chart initiation. LEACH is built on the principle that a selected node as a $\mathrm{CH}$ should not get reselected every time. A sensor node defines a random value between 0 and 1 used to choose the $\mathrm{CH}$. Th(n) represents the threshold value and is evaluated by (1) [15]:

$$
\operatorname{Th}(n)=\left\{\begin{array}{lr}
\frac{\mathrm{p}}{1-\mathrm{p}\left(\mathrm{r} \bmod \mathrm{p}^{-1}\right)} & \text { if } \mathrm{n} \in \mathrm{GN} \\
0 & \text { otherwise }
\end{array}\right.
$$




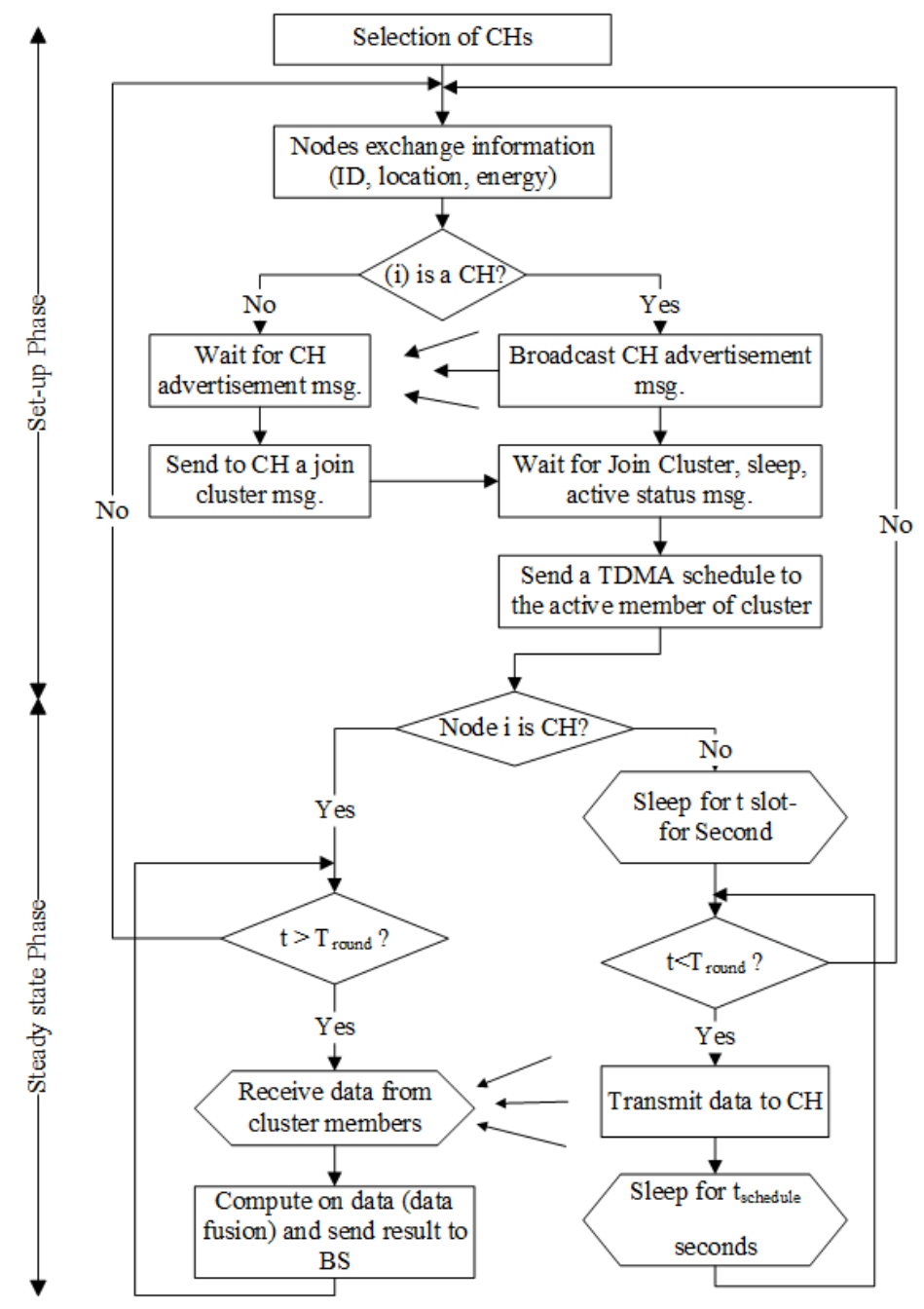

Figure 1. LEACH Protocol

where the $\mathrm{CH}$ probability is represented by (p) in the round number (r), and GN represents the set of nodes that have not been $\mathrm{CH}$ in the last $1 / \mathrm{p}$ rounds. If the selected random value by a specific node is less than $\mathrm{Th}(\mathrm{n})$, then this node will be selected as $\mathrm{CH}$ for the actual round. After selecting the $\mathrm{CH}$, the information about the new $\mathrm{CH}$ is sent to all cluster nodes by a Carrier Sense Multiple Access message (CSMA). This small size message includes the node's ID and a header that specifies the type of message as an announcement message. All WSN nodes receive these announcements originated from different candidate $\mathrm{CHs}$ with different signal strengths. The allocation of a specific node to a specific cluster is made by a decision from the node itself regarding the power intensity of the received announcement. By a CSMA-MAC message, the previous allocation is transmitted back to the selected $\mathrm{CH}$ by sending a Join-Request message back to the $\mathrm{CH}$ as presented in the first part of Figure 1.

- Steady state stage: -

The second part of Fig. 1 shows that this stage can be subdivided into three sub-stage which are the transmission of data from a different location to $\mathrm{CH}$, data aggregation and then data transmission to BS. A TDMA timetable is created by $\mathrm{CH}$ in each cluster, and a random 
CSMA schedule is distributed to all cluster nodes. Each node uses its TDMA slot (schedule) for information routing to $\mathrm{CH}$ of its cluster. To improve the conservation of the nodes' energy, the protocol switches off all passive nodes of the cluster where only the active node and $\mathrm{CH}$ are still switching on. $\mathrm{CH}$ receives all data from various origin, aggregates data and possibly compress it, and transmits the fused data to BS. The most energy consumption happens in the present steady-state stage. Note that the value of $\mathrm{T}_{\mathrm{r}}$ in Fig. 1 represents the round time in sec. Thus, this stage consumes larger time than the setup stage. The procedures included in both stages are repeated periodically from the $\mathrm{CH}$ selection to data received by the BS. The LEACH begins with the $\mathrm{CH}$ selection in the setup stage and finishes by transmitting collected information from various nodes to the BS in the steady-state stage.

\section{B. ABC Protocol}

Artificial Bee Colony (ABC) is an optimization algorithm introduced by Karaboga in 2005. It was inspired by the intelligent behavior of honeybees. ABC, by its simplicity, can be compared to Particle Swarm Optimization (PSO) and Differential Evolution (DE) algorithms. It utilizes only common control parameters like colony size and maximum cycle number. ABC provides a population-based investigation procedure in which individuals named foods positions are adjusted by the artificial bees (with time) and the bee's goal is to find out the places of food sources with high nectar as presented in Fig. 2 [11]. Three bee classes in the ABC are scouts, employed, and onlookers' bees. One bee symbolizes a position in the search space. Based on the bees' experience, artificial bees select their source of food and adapt their next positions. Scouts fly and select the food sources at random without utilizing learned experience. If the nectar quantity of a newly discovered source is greater than the previous one in their memory, bees save the new position and omit the previous. Thus, $\mathrm{ABC}$ integrates local search mode carried out by employed and onlooker bees, with global search modes, managed by onlookers and scouts, and tries to balance exploration and exploitation process. The application of ABC to the WSN routing for a network composed of $\mathrm{n}-\mathrm{CH}$ sensors, gives a dimension of the bees fly in the search space equal to (n).

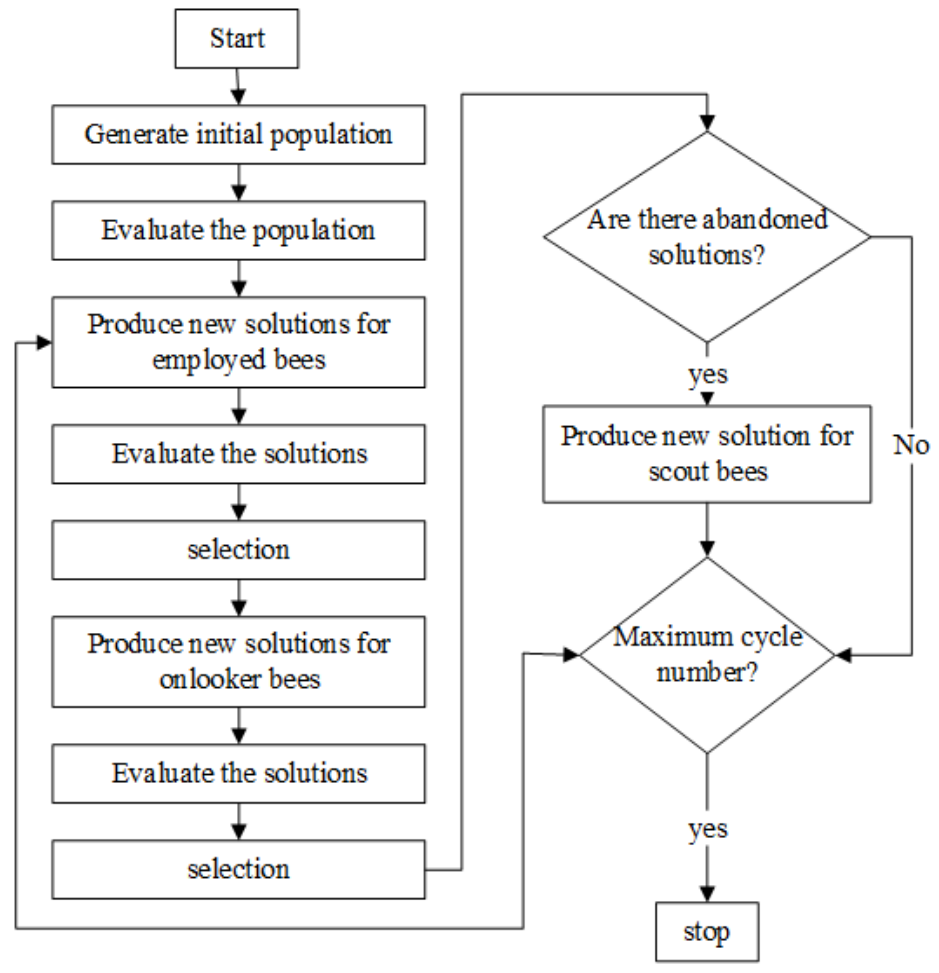

Figure 2. ABC Protocol 
$\mathrm{CHs}$ are located by bees and onlooker bees still waiting on "the dance area" to select the food source. Some sources of food with prior knowledge are visited by the second type of bee which is the employed bee. Finally, a scout is a bee that performs an arbitrary search for food sources. The optimal solution to the issue is represented by the placement of the new source of food. The fineness of the associate problem solution is the nectar quantity of the founded sources. In the $\mathrm{ABC}$ algorithm, food sources are allocated one-to-one to employed bee where the initial sources are arbitrary generated as starting sources. The best food source location is updated in (2) by inspecting the nearby food source by every employed bee in the network. Bee evaluates the quantity of the food where it moves toward the new one in the case of an improvement in the quantity otherwise it keeps working on the present source of food [20].

$$
V_{i j}=X_{i j}+\theta_{i j}\left(X_{i j}-X_{k j}\right)
$$

Where $\theta$ is a random number between $(-1,1), V_{i}$, is a candidate solution, $X_{i}$ is the current solution and $X_{k}$ is a neighbor solution and $j \in\{1,2,3, \ldots, D\}$ is a randomly chosen index where $D$ is the dimension of the solution vector. After accomplishing the search, employed bees broadcast the information concerning the location of the newly discovered food sources with onlookers. The nectar quantity of the discovered source is estimated by (3), and the information is used to select the source by a probability which is the function of the nectar quantity.

$$
\mathrm{P}_{\mathrm{i}}=\frac{\mathrm{fit}_{\mathrm{i}}}{\sum_{\mathrm{n}=1}^{\mathrm{SN}} \mathrm{fit}_{\mathrm{n}}}
$$

where the food source location is represented by (i), $\mathrm{SN}$ defines the employed number (equal to sources number), and fit $t_{i}$ represents the efficiency rate related to the nectar quantity of the solution (i). This selection is performed by the onlookers based on collected information by employed bees. New sources are selected which correspond to $\mathrm{CH}$ in our routing problem by the previous process as roulette wheel selection method. At the moment that food sources have been selected by onlookers one-to-one, followed by a new search of the new nearest source, and then evaluated the nectar quantity of the new source. Onlooker and employed bees can be converted to scout bees if their relative sources of food are consumed. Consumed sources are labeled as deserted and employed bees allocated to these sources are converted to be scout bees. A new possible solution calculated by the scout bee where deserted location is symbolized by $\mathrm{X}_{\mathrm{i}}$ :

$$
X_{i}^{j}=X_{\text {min }}^{\mathrm{j}}+\operatorname{rand}(0,1)\left(X_{\max }^{\mathrm{j}}-X_{\text {min }}^{\mathrm{j}}\right)
$$

\section{Simulation Results}

A 20-node network with 5 clusters is simulated using MATLAB. The initial energy is considered to be 0.1 Joules. The results of the 20 -node WSN are presented in Fig. 3. The topology of the case study is randomly distributed by the program as presented in Fig. 3-a. Fig. 3-b shows a comparison of the residual energy using $\mathrm{ABC}$ and $\mathrm{LEACH}$ protocols for 1500 iterations (rounds). The two curves start from the same point (energy= 0.1 Joules), but after approximately (500) rounds, the residual energy in the case of LEACH protocol is disappeared while in the ABC the energy continuously exists which is better than LEACH protocol. At the end of the 1500 rounds, the $\mathrm{ABC}$ have $0.011275 \mathrm{~J}$ whereas in the case of LEACH only $0.000097 \mathrm{~J}$ remain (y-axis presents the energy, but values are multiplied by (100) time for clarity). The residual energy is directly reflected on the alive nodes as shown in Fig. 3-c where after 1500 rounds the alive nodes of $\mathrm{ABC}$ are (9) nodes while in the case of using LEACH protocol only one (1) node remains alive. The number of received packets depends on alive nodes as in Fig. 3-d, where in the case of ABC a number of 9030400 packets is received while for LEACH the number is limited to only 3131200 yielding an improvement of $288 \%$. Previous results are confirmed by a 40 -node WSN with five clusters where the simulation results are presented in Fig. 4. A comparison of the residual energy using $\mathrm{ABC}$ and $\mathrm{LEACH}$ protocols for this network is outlined for the same number of rounds (1500) in Fig. 4-a. The two curves start from the same point (energy=0.2 Joules), but after (870) rounds the residual energy of the $\mathrm{LEACH}$ protocol already vanishes while in the $\mathrm{ABC}$, it is equal to $0.074844 \mathrm{~J}$ ( $37 \%$ of the original energy). The $\mathrm{ABC}$ has maintained better performance than the LEACH protocol up till the end of round 1500 where the ABC stills keeping $0.01955 \mathrm{~J}(9.7 \%$ of 
the original energy). However, the residual energy is directly reflecting the alive nodes as in Fig. 4-b, where after 870 rounds the alive nodes of LEACH are zero whereas in the ABC all nodes still alive. At the end of the 1500 rounds, 4 nodes still alive, which proves again that the $A B C$ shows better performance.

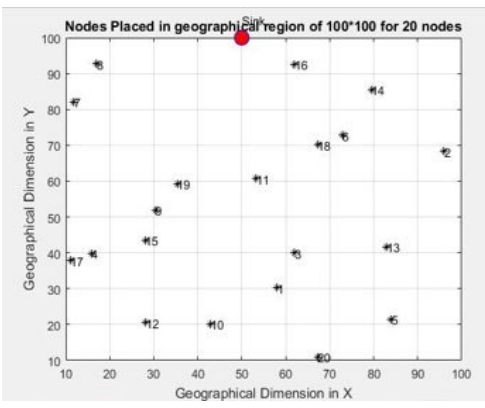

(a)- Network topology

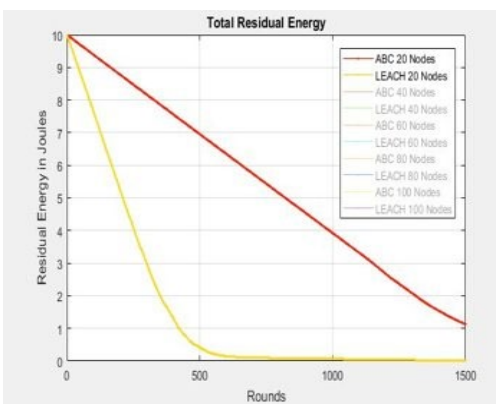

(b)- Residual energy

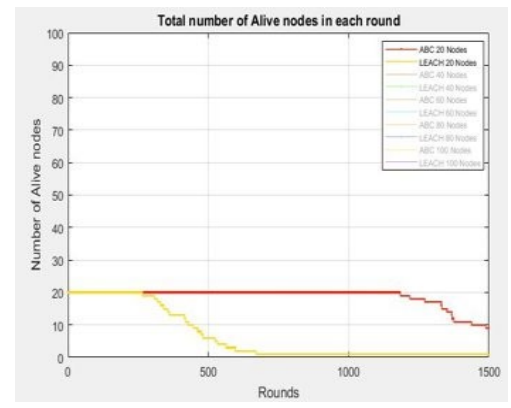

c)- Alive node

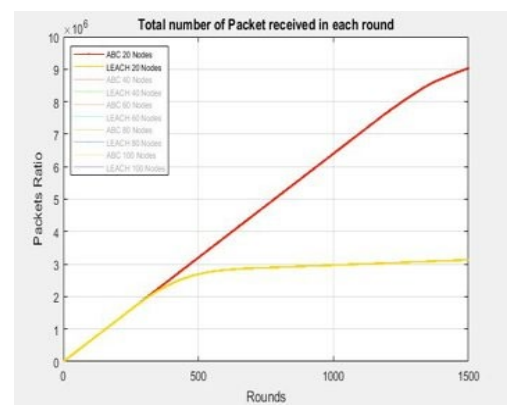

(d)- Packet number

Figure 3. 20-node WSN 


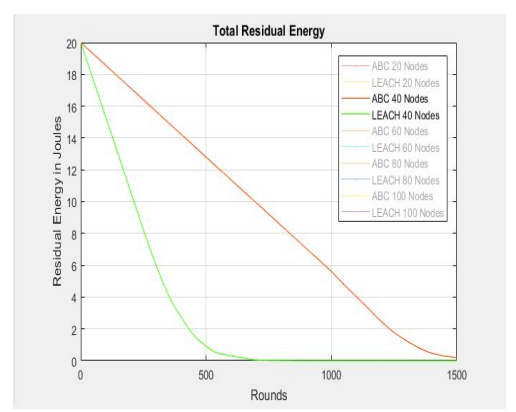

a)- Residual energy

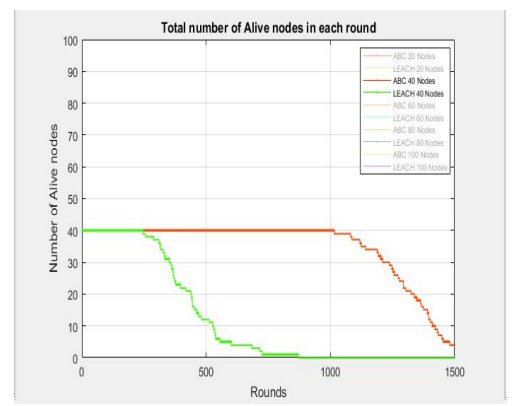

(b)- Alive node

Figure 4. 40-node WSN

\section{Conclusion}

This work is concerned with the improvement of routing protocol performance of the dynamic clustering routing protocols in WSN. A study is conducted on the evaluation of the performances of routing algorithm- based LEACH protocol. The routing algorithm is improved by using ABC optimization protocol as a tool for a dynamic $\mathrm{CH}$ selection. Residual energy collected from the simulation of $\mathrm{LEACH}$ alone and compared to $\mathrm{LEACH}$ with $\mathrm{ABC}$ for all scenarios shows a significant improvement in the case of ABC-based LEACH.

The simulation also proved the improvement made by the use of $\mathrm{ABC}$ on the dead node number, network lifetime, and received packets. Many scenarios were implemented to evaluate the improvement made by the $\mathrm{ABC}$ to the energy consumption. The results illustrate the superiority of the ABC-based LEACH compared to LEACH protocol alone. The improvement becomes more pronounced for networks with a high number of clusters.

\section{References}

[1]. V. Dhiman, M. Kumar, and A. K. Sharma, "A Radical Study of Energy Efficient Hierarchical Cluster-Based Routing Protocols for WSN," Inter. J of Elec. Engineering and Informatics, vol. 12(3), pp. 445-469, 2020.

[2]. K. Sohraby, D. Minoli, and T. Znati, Wireless sensor networks: technology, protocols, and applications. John Wiley \& Sons, 2007.

[3]. A. Kumar, M. Zhao, K. Wong, Y. L. Guan, and P. H. J. Chong, "A comprehensive study of IoT and WSN MAC protocols: research issues, challenges and opportunities," IEEE Access, vol. 6, pp. 76228-76262, 2018.

[4]. S. K. Yang, Y. M. Shiou, Z. Y. Su, I. H. Liu, and C. G. Liu, “An authentication information exchange scheme in WSN for IoT applications," IEEE Access, vol. 8, pp. 9728-9738, 2020.

[5]. S. A. Haque, M. Rahman, and S. M. Aziz, "Sensor anomaly detection in wireless sensor networks for healthcare," Sensors, vol. 15, pp. 8764-8786, 2015. 
[6]. N. N. Malik, W. Alosaimi, M. I. Uddin, B. Alouffi, and H. Alyami, "Wireless sensor network applications in healthcare and precision agriculture" J. of Healthcare Eng., pp. 19, 2020.

[7]. Z. G. Wan, Y. K. Tan, and C. Yuen, "Review on energy harvesting and energy management for sustainable wireless sensor networks," 13th IEEE Inter. Conf. on Comm. Technology (ICCT), pp. 362-367, 2011.

[8]. Hajer F. Fadh, Musaria K. Mahmood, Osama, Al-Omari, "A comprehensive analysis of energy dissipation in LEACH protocol for wireless sensor networks," $18^{\text {th }}$ Int. Multi-Conf. on Systems, Signals \& Devices, pp. 53- 57, Tunisia, 2021.

[9]. C. Li, H. Zhang, B. Hao, and J. Li, "A survey on routing protocols for large-scale wireless sensor networks," Sensors, vol. 11(4), pp. 3498-3526, 2011.

[10]. R. I. Tandel, "LEACH protocol in wireless sensor network: A Survey,” Int. J. of Computer Science and Information Technologies, vol. 7(4), pp. 1894-1896, 2016.

[11]. D. Karaboga, S. Okdem, and C. Ozturk, "Cluster based wireless sensor network routing using artificial bee colony algorithm,” Wireless Networks, vol. 18(7), pp. 847-860, 2012.

[12]. A. Alabass, K. Elleithy, and A. Razaque, "Dynamic cluster head node election model over wireless sensor networks," $29^{\text {th }}$ Int. Conf. on Comp. and Their Appl., March 2014.

[13]. A. T. Nuray and S. M. Daraghma, "A new energy efficient clustering-based protocol for heterogeneous wireless sensor networks. J. of Elec. \& Electronics. Sys., vol.4(3), pp. 1-7, 2015.

[14]. Lakshmeesha P, and Shiva Murthy G, "Dynamic cluster head selection mechanism for wireless sensor networks," Int. J. of Eng. and Computer Science, vol. 5(9), pp. 1788417888,2016

[15]. R. K. Gill, P. Chawla, and M. Sachdeva, "Study of LEACH routing protocol for wireless sensor networks," Int. Conf. on Comm., Computing \& Systems, 2016.

[16]. G. S. Arumugam and T. Ponnuchamy, "EE-LEACH: development of energy-efficient LEACH Protocol for data gathering in WSN," EURASIP J. Wireless Comm. Net., vol. (1), pp. 1-9, 2015

[17]. R. Sinde, F. Begum, K. Njau, and S. Kaijage, "Lifetime improved WSN using enhancedLEACH and angle sector-based energy-aware TDMA scheduling," Cogent Eng., vol. 7(1), pp. 1-21, 2020.

[18]. D. Jia, H. Zhu, S. Zou, and P. Hu, "Dynamic cluster head selection method for wireless sensor network. IEEE Sensors J., vol. 16(8), pp. 2746-2754, 2016.

[19]. N. N. A. Qubbaj, A. Abu Taleb, and W. Salameh, "LEACH Based protocols: a survey," Adv. in Sci., Tech. and Eng. Sys. J., vol. 5(6), pp. 1258-1266, 2020.

[20]. D. Karaboga, An idea based on honey bee swarm for numerical optimization., Technical Report - TR06, 2005

[21]. D. Karaboga and C. Ozturk, "A novel clustering approach: Artificial Bee Colony (ABC) algorithm," Appl. Soft Comp., vol. 11(1), pp. 652-657, 2011.

[22]. A. Yadav, S. Kumar, and S. Vijendra, "Network life time analysis of WSNs using particle swarm optimization," Procedia Comp. Sci., vol. 132, pp. 805-815, 2018.

[23]. S. Famila, A. Jawahar, A. Sariga, and K. Shankar, "Improved artificial bee colony optimization based clustering algorithm for smart sensor environments," Net. and Appl., vol. 13, pp. 1071-1079, 2020.

[24]. P. S. Mann, and S. Singh, "Artificial bee colony metaheuristic for energy-efficient clustering and routing in wireless sensor networks," Soft Computing, vol. 21(22), pp. 66996712, 2017.

[25]. D. Kajaree and R. Behera, "A survey on web Crawler approaches," Int. J. of Innov. Res. in Comp. and Comm. Eng., vol. 5(2), pp. 1302-1309, 2017.

[26]. S. Jabbar et al., "Analysis of factors affecting energy aware routing in wireless sensor network," Wireless Comm. and Mobile Computing, 2018. 
[27]. Wendi R. Heinzelman, A. Chandrakasan, and H. Balakrishnan," Energy-efficient communication protocol for wireless microsensor networks," $33^{\text {rd }}$ Hawaii Int. Conf. on System Sciences, pp. 1-10, 2000.

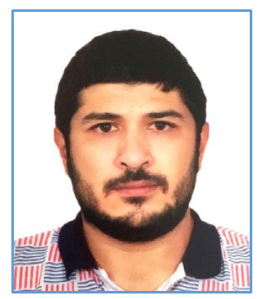

Sulaiman M. Karim received his MS degree in Computer Engineering, Erciyes University in 2019. He is actually a PhD student in Karabuk University. His research interests include clustering and optimization in wireless sensor networks, Blockchain and IoV security.

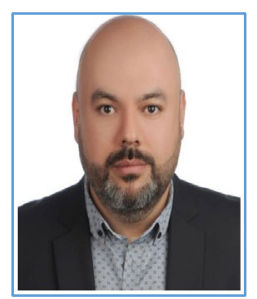

Celal Öztürk received the doctorate in Computer Engineering from the Erciyes University. He received his MS degree from Electrical and Computer Engineering, Rutgers University and his BSc degree in Computer Engineering from Erciyes University. From 2007 to 2011, he was a Lecturer at the Department of Computer Engineering, Erciyes University and he is currently working as an Associated Professor. His research interests include training neural networks, clustering and optimization in wireless sensor networks and bioinformatics.

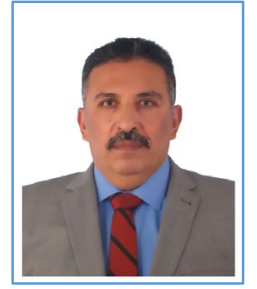

Musaria K. Mahmood received the doctorate in Communication \& Electronics Engineering from Al-Nahrain University. He received his MS degree in Communication \& Electronics Engineering, Al-Nahrian University and his BSc degree in Electronics Engineering from ENSIETA (France). From 2003 to 2016, he was a Lecturer at the Department of Electrical Engineering, Tikrit University (Iraq) and he is currently working as an Assistant Professor in Istanbul Gelisim University. His research interests include network reliability, clustering, and WSN. 